\section{Psychogenic sneezing}

Sir,

Sneezing is considered to be a physiological defense mechanism against nasal mucosal irritation due to allergy, infection or local pathology. Unusual triggers of sneezing include light, full stomach, sexual ideation and orgasm. ${ }^{[1]}$ Psychogenic sneezing is a relatively rare entity, first described by Shilkret in 1949. ${ }^{[2]}$ Since then it has been accepted as a clinical entity, commonly considered as a differential in chronic intractable cases of sneezing.

An adolescent girl belonging to lower socio-economic status from a rural background was brought by her parents with periodic bouts of sneezing of 15 days duration. She was found to have paroxysms of three sneezes at a time which used to get repeated after a short delay, persisting for 2-3 hours especially in late evening. Her preparatory augmented inspiration phase was barely observable which was followed by relatively mild burst of expiratory thrust, unusual for a normal sneeze reflex. Episodes happened with eyes open and were associated with anxiety and mild depressive features. Symptoms used to disappear while conversing, eating and sleeping. There was no associated nasal discharge, rhinorrhea, lacrimation, photophobia, phonophobia, earache, earwax, facial pain or toothache. She was referred for psychiatric evaluation by an ENT surgeon. Clinical examination and vital parameters were found to be within normal limits. X-ray for paranasal sinuses, complete blood count and chest X-ray were normal. ENT surgeon had put her on a trial of antihistaminic and nasal decongestants, without any improvement. On detailed psychiatric evaluation, it was found that she got married 1 month back. She reported that she was unaware of sex and was fearful about physical intimacy with her husband. And after few days of intimacy, she developed the above-described symptoms. Timing of these symptoms approximately matched the usual period of physical intimacy. The couple started abstaining from sex. There was no past significant medical or surgical history including allergy. She has a younger sibling with symptoms suggestive of bronchial asthma who used to get more parental attention while she was made to do all household work. Her birth and developmental history was unremarkable. Premorbidly she was shy, socially inhibited and had a slow to warm temperament. Considering her sneezing episodes had no physical basis with history of recent marriage and ignorance about sexual activities (sexual conflict) and her pampered younger sibling being a model for her apparent physical symptoms, a diagnosis of psychogenic sneezing (dissociative disorder) was considered. Low-dose benzodiazepines were administered for immediate anxiety relief along with tablet sertraline $25 \mathrm{mg}$, gradually raised to $100 \mathrm{mg}$. Psychotherapeutic interventions were administered in the form of relaxation and sex education. Family members were explained about her condition and their support was ensured. Over a period of 1 week, she experienced significant improvement in her symptoms. She was discharged and was temporarily taken back to her parents' home after 10 days. On follow up after 2 weeks, she was completely symptom free and was happy with her husband.

The above case matches the general criteria involved in diagnosing a case of intractable paroxysmal psychogenic sneezing. ${ }^{[3]}$ Further, several interesting cases of sneezing, rhinitis and cough have also been reportedly associated with coitus and they have been quite suggestively termed as suffering from "Honeymoon Rhinitis." ${ }^{[1,4]}$ Our case is different with the symptom originating few days after initiation of physical intimacy and later episodes occurred without any physical contact. All the more, there was no associated rhinitis or cough. The mild quality of expiratory thrusts with minimal preparatory inspiratory phase, as described earlier, suggests an atypical picture. Considering the absolute lack of sexual knowledge and the terror associated with the initial few acts, she could have unconsciously resorted to sneezing as a form of help-seeking behavior. Her pampered young brother with episodes of asthma and allergic sneezing could have become a model for attracting attention (secondary gain) for this shy, socially inhibited young lady. The episodes led to stoppage of coital act which can be considered as her primary gain, thus satisfying all the required parameters for establishing a diagnosis of dissociative disorder. A biological explanation could also be considered in this case with unpleasant sexual ideation leading to autonomic arousal with probable "parasympathetic summation."[1] This original hypothesis by Bhutta and Maxwell did not specify about the nature of sexual ideation in their subjects. In our case, the sexual ideation was distinctly unpleasant, thereby potentially adding another domain to the existing hypothesis. Further, her sneezing can also be explained through the emotional centers of respiratory control involving pre-frontal cortex, medial orbito-frontal cortex, periaqueductal gray area and nucleus retroambiguus. ${ }^{[5]}$ Administration of sertraline and benzodiazepines could have helped in modulating the autonomic nervous system tone along with relieving anxiety. ${ }^{[6,7]}$ Further, relaxation training and sex education for the patient along with ensuring family support would have aided the process of recovery.

This case yet again demonstrates the intriguing ways of presentation of dissociative disorders, challenging the era of biological psychiatry. It also highlights the 
still existing veil of shame and embarrassment covering the sensitive yet important topic of sex among families. Adequate parent-child communication along with imparting family life education in schools should be addressed upon. ${ }^{[8]}$ Further, always maintaining a margin of suspicion is essential in all branches of medicine for quick identification and treatment of psychogenic (dissociative) disorders with least economic and psychological burden as demonstrated in our case.

Ajish G. Mangot, Satyakant Trivedi, Alaknanda Pandey, Desiree Saimbi, Vaibhav Dubey

Department of Psychiatry, People's College of Medical Sciences and Research Centre, Bhopal, Madhya Pradesh, India

Address for correspondence: Dr. Ajish G. Mangot,

Psychiatry OPD-7, C-Block, People's College of Medical Sciences and Research Centre, Bhanpur, Bhopal - 462037, Madhya Pradesh, India. E-mail: dr.ajish@outlook.com

\section{References}

1. Bhutta MF, Maxwell H. Sneezing induced by sexual ideation or orgasm: An under-reported phenomenon. J R Soc Med 2008;101:587-91.
2. Shilkret HH. Psychogenic sneezing and yawning. Psychosom Med 1949;11:127.

3. Bhatia MS, Khandpal M, Srivastava S, Khandpal M. Intractable psychogenic sneezing: Two case reports. Indian Pediatr 2004;41:503-5.

4. Monteseirin J, Camacho MJ, Bonilla I, Sánchez-Hernández C, Hernández M, Conde J. Honeymoon rhinitis. Allergy 2001;56:353-4.

5. Holstege G, Beers CM, Subramanian HH. The Central Nervous System Control of Respiration. Oxford, Elsevier; 2014. p. 379-406.

6. Shores MM, Pascualy M, Lewis NL, Flatness D, Veith RC. Short-term sertraline treatment suppresses sympathetic nervous system activity in healthy human subjects. Psychoneuroendocrinology 2001;26:433-9.

7. Agelink MW, Majewski TB, Andrich J, Mueck-Weymann M. Short-term effects of intravenous benzodiazepines on autonomic neurocardiac regulation in humans: A comparison between midazolam, diazepam, and lorazepam. Crit Care Med 2002;30:997-1006.

8. Tripathi N, Sekher TV. Youth in India ready for sex education? Emerging evidence from national surveys. PLoS One 2013;8:e71584.

\begin{tabular}{|l|l|}
\hline \multicolumn{2}{|c|}{ Access this article online } \\
\hline Quick Response Code: & Website: \\
\hline & www.ruralneuropractice.com \\
\cline { 2 - 2 } & \\
\hline & \\
\hline
\end{tabular}

